\title{
Growth phase-dependent responses of an in vitro gut microbiome to metformin
}

\author{
Zikai Hao ${ }^{1,2,3}$, Leyuan $\mathrm{Li}^{2}$, Zhibin Ning ${ }^{2}$, Xu Zhang ${ }^{2}$, Janice Mayne ${ }^{2}$, Kai Cheng ${ }^{2}$, Krystal \\ Walker $^{2}$, Hong Liu ${ }^{1,3_{*}}$, Daniel Figeys ${ }^{1,2_{* *}}$
}

${ }^{1}$ Beijing Advanced Innovation Centre for Biomedical Engineering, Beihang University, Beijing, China

${ }^{2}$ Department of Biochemistry, Microbiology and Immunology, Ottawa Institute of Systems

Biology, Faculty of Medicine, University of Ottawa, Ottawa, Canada.

${ }^{3}$ School of Biological Science and Medical Engineering, Beihang University, Beijing, China

* Correspondence to Hong Liu: e-mail: $\underline{\text { h64 @ buaa.edu.cn }}$

**Correspondence to Daniel Figeys: e-mail: dfigeys@uottawa.ca 


\section{Abstract}

In vitro gut microbiota models are often used to study drug-microbiome interaction. Similar to culturing individual microbial strains, the biomass accumulation of in vitro gut microbiota follows a logistic growth curve. Current studies on in vitro gut microbiome responses introduce drug stimulation during different growth stages, e.g. lag phase or stationary phase. However, in vitro gut microbiota in different growth phases may respond differently to same stimuli.

Therefore, in this study, we used a 96-deep well plate-based culturing model (MiPro) to culture the human gut microbiota. Metformin, as the stimulus, was added at the lag, log and stationary phases of growth. Microbiome samples were collected at different time points for optical density and metaproteomic functional analysis. Results show that in vitro gut microbiota responded differently to metformin added during different growth phases, in terms of the growth curve, alterations of taxonomic and functional compositions. The addition of drugs at log phase leads to the greatest decline of bacterial growth. Metaproteomic analysis suggested that the strength of the metformin effect on the gut microbiome functional profile was ranked as lag phase $>\log$ phase $>$ stationary phase. Our results showed that metformin added at lag phase resulted in a significantly reduced abundance of the Clostridiales order as well as an increased abundance of the Bacteroides genus, which was different from stimulation during the rest of the growth phase. Metformin also resulted in alterations of several pathways, including energy production and conversion, lipid transport and metabolism, translation, ribosomal structure and biogenesis. Our results indicate that the timing for drug stimulation should be considered when studying drugmicrobiome interactions in vitro.

\section{Introduction}

A major recent paradigm shift in medicine is the recognition of the important roles that the gut microbiome plays in drug therapy ${ }^{1,2}$. The activity of drugs is usually dictated not only by the host, but often also by the gut microbiome. Evidences suggest that drugs can affect the functions and composition of the gut microbiome $\mathrm{e}^{3-5}$. Increasing studies are focused on drug-microbiome interactions $^{1,6}$. In vitro gut microbiome culturing models are rapid, and cost-efficient approaches for studying drug-microbiome interactions and are increasingly applied to microbiome studies ${ }^{7-}$ ${ }^{11}$. For example, the SHIME reactor (Simulator of the Human Intestinal Microbial Ecosystem) ${ }^{11,}$ 
${ }^{12}$, and the M-SHIME ${ }^{13}$ have been used to study drug effects on microbiome; Jalili-Firoozinezhad et al. ${ }^{7,14}$ have developed a microfluidic intestine-on-a-chip to study microbiome-related therapeutics, probiotics and nutraceuticals; Shah et al. ${ }^{8}$ have also presented a modular, microfluidics-based model (HuMiX, human-microbial crosstalk), which allows co-culture of human and microbial cells under conditions representative of the gastrointestinal human-microbe interface. Wu et al. ${ }^{10}$ have used an in vitro simulated human intestinal redox model (SHRIM) to investigate metformin-microbiota interactions directly. We have previously developed a 96-deep well plate-based culturing model (MiPro) facing high-throughput drug screening ${ }^{15}$.

Previous studies have added drug stimulation to the in vitro gut bacteria at various points during the growth phases of the gut microbiome. For example, Maier et al. ${ }^{16}$, Li et al. ${ }^{15}$ and Wang et al. ${ }^{17}$ studied microbial responses to drugs by inoculating bacterial strains or fecal inoculum into culture mediums containing drugs. Wu et al. ${ }^{10}$ used the SHRIM to explore the effect of metformin on a stabilized gut microbial community in vitro, and added the metformin after one week of stabilization in SHRIM. Similarly, with the SHIME models, compound are also added following 1 2 weeks of stabilization ${ }^{11,12}$. Notably, bacteria can respond differently to environmental stimulations during different points in the growth phases. Early studies on the mechanism of action of penicillin showed that the bactericidal activity of $\beta$-lactam antibiotics depends on bacterial growth ${ }^{18,19}$. This is best illustrated by the inability of penicillin to kill nongrowing bacteria in contrast to the rapid killing and lysis of the same cells during exponential growth $^{20}$. We therefore postulated that the in vitro gut microbiome may also functionally respond differently to drugs added at different points in the growth phases, which, to the best of our knowledge, has not been reported yet.

In this study, we cultured a human gut microbiome using the Mipro model and added metformin at the lag, log and stationary phases of growth. Cultured samples were collected overtime for biomass and metaproteomic analyses. By analyzing growth curves and taxon-specific functional profiles, we demonstrated that adding metformin at log phase led to the greatest decline of bacterial growth while at the lag phage it induced the greatest changes on the functional profile. This highlights the importance of drug stimulation time in the investigation of drug-microbiome interactions. 


\section{Results}

\section{Determination of the microbiome growth curve}

In this study, we hypothesized that drugs added at different growth phase of an in vitro microbiota may have different effects (Figure 1A). In order to determine the time points (i.e. lag, $\log$ and stationary phases) of drug stimulation, we first measured the growth curve of the in vitro gut microbiome. Briefly, a human gut microbiome was cultured in each well of a 96-well plate using MiPro ${ }^{15}$. Samples were taken at 15 time points in triplicates from 0-48 hours of culturing (Figure 1B). Optical density of each sample was measured at $595 \mathrm{~nm}\left(\mathrm{OD}_{595}\right)$, as a proxy of microbial biomass. The growth curve of the in vitro gut microbiome was calculated by fitting a logistic growth model to the data from each time point(Figure.1B). The microbial biomass $N t$ at time $t$ was described by the following logistic equation:

$$
N t=\frac{0.466}{1+41.36 e^{-0.778 t}}
$$

From Eq. (1) we calculated that the maximum possible microbial biomass $(K)$ was 0.466 , and the intrinsic growth rate of the microbiome $(r)$ was 0.778 . At $4.8 \mathrm{hr}$, the microbiome reached the maximum growth rate, i.e. the microbial biomass reached $1 / 2 K$. The microbiome achieved stationary growth at about $12 \mathrm{hr}$.

We then tested the responses of the microbiome to metformin at three different times point representing different growth phases, i.e. $0 \mathrm{hr}$ - lag phase (Lag), $5 \mathrm{hr}$ - log phase (Log) and $12 \mathrm{hr}$ - stationary phase (STA). Briefly, metformin was added at a final concentration of $7.6 \mathrm{mM}$ into designated wells at the three growth phases $^{15}$ (Figure 1C). Following each metformin treatment, samples were collected in triplicates over time for optical density and metaproteomic analyses (Figure 1C). 

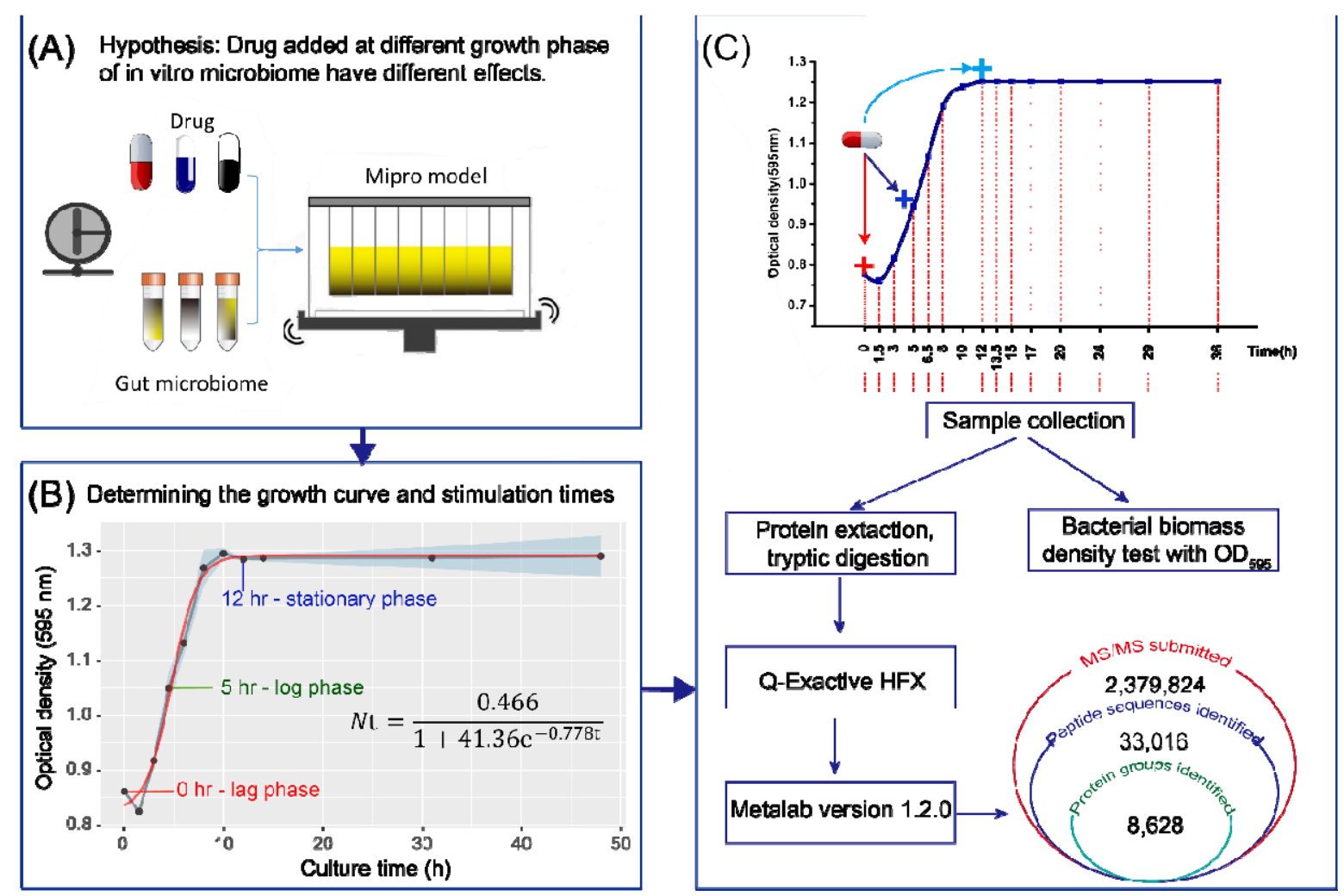

Figure.1 Experimental hypothesis and workflow. (A) Experimental hypothesis. (B) In vitro growth of the human gut microbiome in 48 hours. Black dots are means ( $\mathrm{n}=3$ individual wells) of the $\mathrm{OD}_{595}$ value of the culture, and blue ribbon stands for standard deviation. Red line is the logistic growth model fitted based on the mean $\mathrm{OD}_{595}$ value. (C) Experimental workflow. Human gut microbiome samples were cultured with metformin added at $0^{\text {th }}$ hour, $5^{\text {th }}$ hour and $12^{\text {th }}$ hour. Then, the samples in 96 -well deep-well plates were collected for microbial biomass and metaproteomics analysis. Peptide and protein identification and quantification, taxonomic profiling, and functional annotation were performed using the automated MetaLab software ${ }^{21}$.

\section{Stimulation at the log phase resulted in the strongest antibacterial action}

To compare the difference in growth curve between different treatment times, t-test and one-way ANOVA were performed. Results show that the biomass of the treatment groups (Lag/Log/STA) was significantly lower than the control group following the addition of metformin (Figure 2A). Moreover, after $20 \mathrm{hr}$, all metformin treatments showed loss of biomass while the control microbiome was still at the stationary phase, indicating that metformin has a significant antibacterial effect. As well, the trajectories of the curves were different between groups with the addition of metformin during the log phase causing a steep decline after 20 hours while the addition during the Lag and STA phases did not cause a significant continuous decline. 


\section{Microbiome functional response to metformin is associated with the microbial growth phase}

We then explored whether adding metformin during the different phases can lead to difference in functional changes in the microbiome. Briefly, metaproteomic analysis was performed on 85 samples collected at different time points (5-7 time points in each group, and triplicates samples at each different time point, Supplementary Table S2). 2,379,824 MS/MS spectra were obtained from all 85 raw files, resulted in 33,016 peptides and 8,628 protein groups (FDR < 1\%; Figure 1C). The average Pearson's correlation coefficient between metaproteomic quality control samples was $0.992 \pm 0.001$ (mean $\pm \mathrm{SD}, \mathrm{n}=3$ ), and the correlation between each group of technical replicates ranged from $0.956 \pm 0.028$ to $0.990 \pm 0.001(n=3$; Supplementary Figure $S 1$ and Table S1). These results indicated high reproducibility of both culturing and metaproteomics approach.

We first looked at the differences in protein expressions between treatment and control groups at each time point and after the same time of stimulation based on label-free quantification (LFQ) protein intensities (FDR-adjusted $P<0.05)$. Different proteins had significant changes in expression at each time points and between treatment groups compared to the control group (Figure 2B) suggesting that the gut microbiome responded differently to metformin added at the three time points. Interestingly, introducing metformin at the lag phase led to a cycle of differentially expressed proteins with a first maximum at 6.5 hours and maximum about every 10-12 hours thereafter. During 24-36 hr, when metformin exerted significant antibacterial effects on the microbial biomass significantly declined (Figure 2A), the number of significantly altered proteins increased again. However, the Lag group had the highest response during the whole growth phase.

We then compared the number of significantly altered protein expression across the growth phases after an equal amount of time (1.5, 5, 1224 hours) following the introduction of metformin (Figure 2C; FDR-adjusted $P<0.05$ ). The result confirmed that the introduction of metformin during different growth phases led to changes in protein expressions in the 
microbiome that remain distinct over time. Interestingly, the highest responses were observed while groups were in the log phase following treatment. After 24 hours of stimulation, the response in all treatment groups increased significantly, likely due to the significant antibacterial effect of metformin at that time.

The addition of metformin at different growth phase also led to alterations of microbiome genera over time (Supplementary Figure S3). Interestingly, the changes in the relative abundance of genera Bacteroides and Fusobacterium showed a cyclic pattern similar to the significantly altered proteins (Figure 2B), which may also explain the cyclic pattern observed in significantly altered proteins. 
(A)

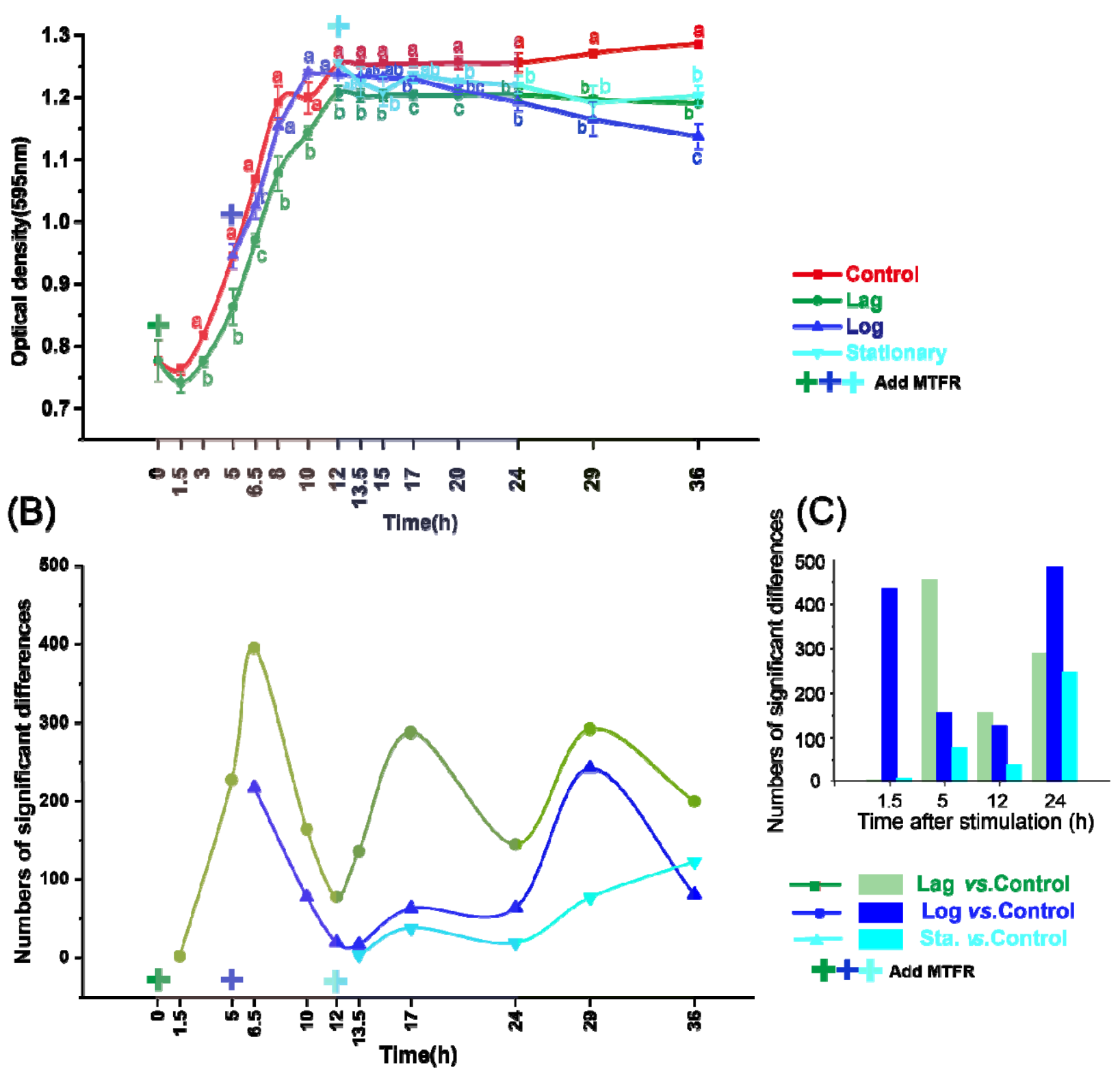

Figure.2 Metaproteomic response to metformin treatment was associated with the growth phase.

To compare the difference in growth curve and metaproteomic profiles between different treatment times, t-test was performed at the time points for 1.5-5 hr (control vs. Lag only), and one-way ANOVA was performed at the time points for 6.5-36 hr. (A) Gut microbiome response differently to metformin on the growth curve when metformin added at different growth phase. Optical density at $595 \mathrm{~nm}$ (OD595) was used as a proxy of microbial biomass. Data were shown as averages and error bars represent SEM; statistically significant differences were shown with 
different letters ( $\mathrm{p}<0.05)$. T-test and one-way ANOVA with Duncan's test was performed. (B) Numbers of significantly altered protein groups between the treatment group and control group changed over time (FDR-adjusted $\mathrm{P}<0.05$ ). (C) Numbers of significantly altered protein groups between the treatment group and control group after the same hours of stimulation (FDRadjusted $\mathrm{P}<0.05)$. T-test was performed.

Stimulation at the lag phase resulted in the strongest metaproteomic response

We further visualized the overall change of protein group intensities using principal component analysis (PCA) (Figure 3A). In most cases, the three groups were differentiated on the PCA score plots. Interestingly, the differentiation was related to the numbers of significantly altered protein groups at the corresponding points (Figure 2B). We further performed multivariate analysis of variance (MANOVA) to statistically compare metaproteomic differences between different groups. Hierarchical clustering was generated based on mahalanobis distances as a result of MANOVA (Figure 3C and Supplementary Figure S2). The MANOVA analysis showed that there were significant differences $(\mathrm{p}<0.05)$ between each treatment and the control at each time point (with exception of STA vs control at 13.5 and $24 \mathrm{hr}$ ). In particular, the PCA and MANOVA analyses suggested that the Lag group had the highest response among the three groups. PCA score plot (Figure 3B) and MANOVA analysis (Figure 3C) based on the last three time points (24, 29 and $36 \mathrm{hr})$ showed a clear separation between control, Lag, Log and STA groups, suggesting that the strengths of metaproteomic responses were ranked as Lag> Log > STA groups. 
(A)
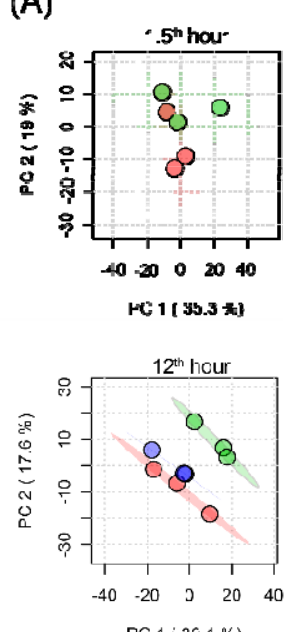

PC 1 i $30.1 \%$ )

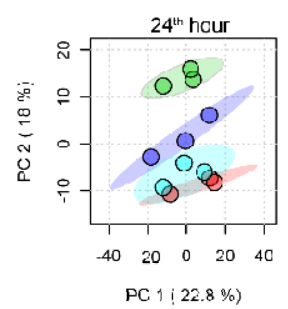

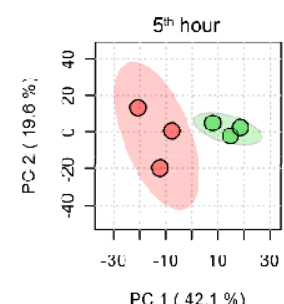

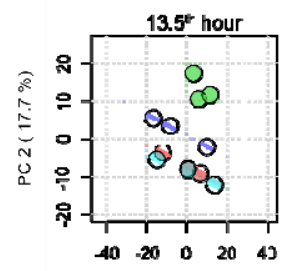

PC 1 (21.8\%)

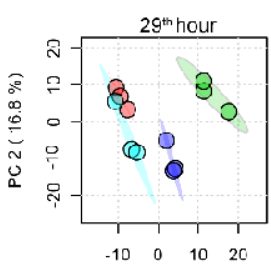

PC $1(20.1 \%)$
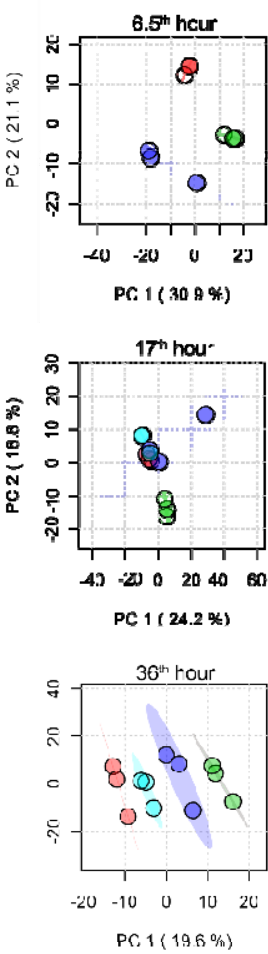

(B)

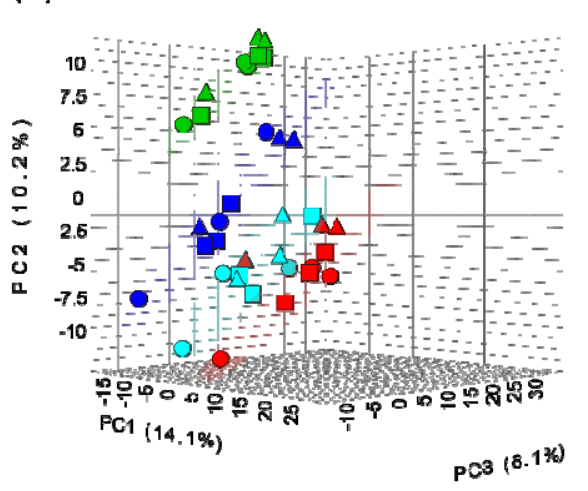

Treatment - Control

Lag

- Log

Stationary

Culture time

$24^{\text {th }}$ hour

$24^{\text {h' hour }}$
(C)

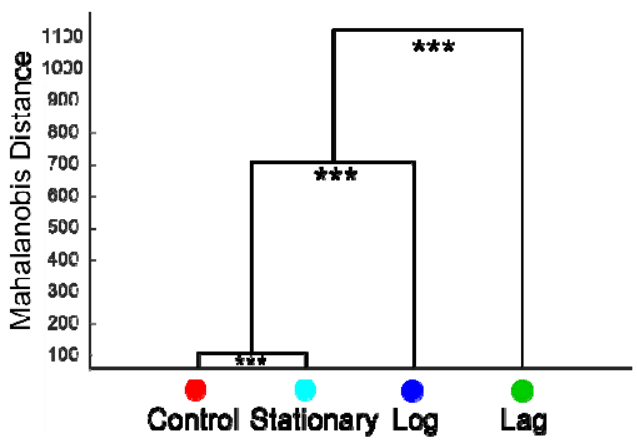

Figure 3. The alteration of gut microbiome in different groups response to metformin over time. (A) Principal component analyses (PCA) scores plots based on protein groups of the samples at the 1.5, 5, $6.5,12,13.5,17,24,29$ and $36 \mathrm{hr}$, respectively. (B) PCA plots based on the protein groups of the all samples during the $24-36 \mathrm{hr}(24,29$ and $36 \mathrm{~h})$. PCA plots showing metaproteomic profiles were different when metformin was added at different growth phases. (C) Clustering of different groups during the 2436 hour based on mahalanobis distances calculated using MANOVA, ***, P $<0.001$. The PCA plots and mahalanobis distances of MANOVA analysis showing strength of metformin effect on gut microbiome functional profiles was ranked as lag phase $>\log$ phase $>$ stationary phase.

A time-series analysis using ANOVA - Simultaneous Component Analysis (ASCA) revealed patterns of protein expression that are time or treatment specifics as well as patterns that depend on both time and treatment simultaneously (interaction), Figure 4A-H. The pattern of "Treatment" or "Time" is the case where no time-treatment group interactions were modeled, only a treatment or time effect in all the groups. The score profiles of the first component $(73.3 \%$ of variability) of the "Treatment" showed that the strength of the metformin effect on the gut microbiome was ranked as Lag group > Log group > STA group > Control group (Figure 2E). The score profiles of the first component (57.23\% of variability) of the "Time" showed a 
negative effect through time for all groups (Figure 2F). The scores of all groups decreased rapidly during the log phase (1.5-12 hr) and gradually stabilized during the stationary phase (13.5-36 hr), which suggested that the strongest metaproteomic response to metformin treatment was associated with the growth phase (Figure $2 \mathrm{~F}$ ). The score profiles of the first two components of the "Interaction" (treatment plus time) described the different behavior for all groups over time (Fig. 2G-H). The score profiles of the first component (23.3\% of variability) identified a marked effect through time for the Lag group which was different from the rest of groups. The Second component (10.35\% of variability) identified a marked effect through time for the Log group which was different from the other groups. Finally, the scores of the STA group were similar to the control group in the score profiles of the "Treatment" and "Interaction".
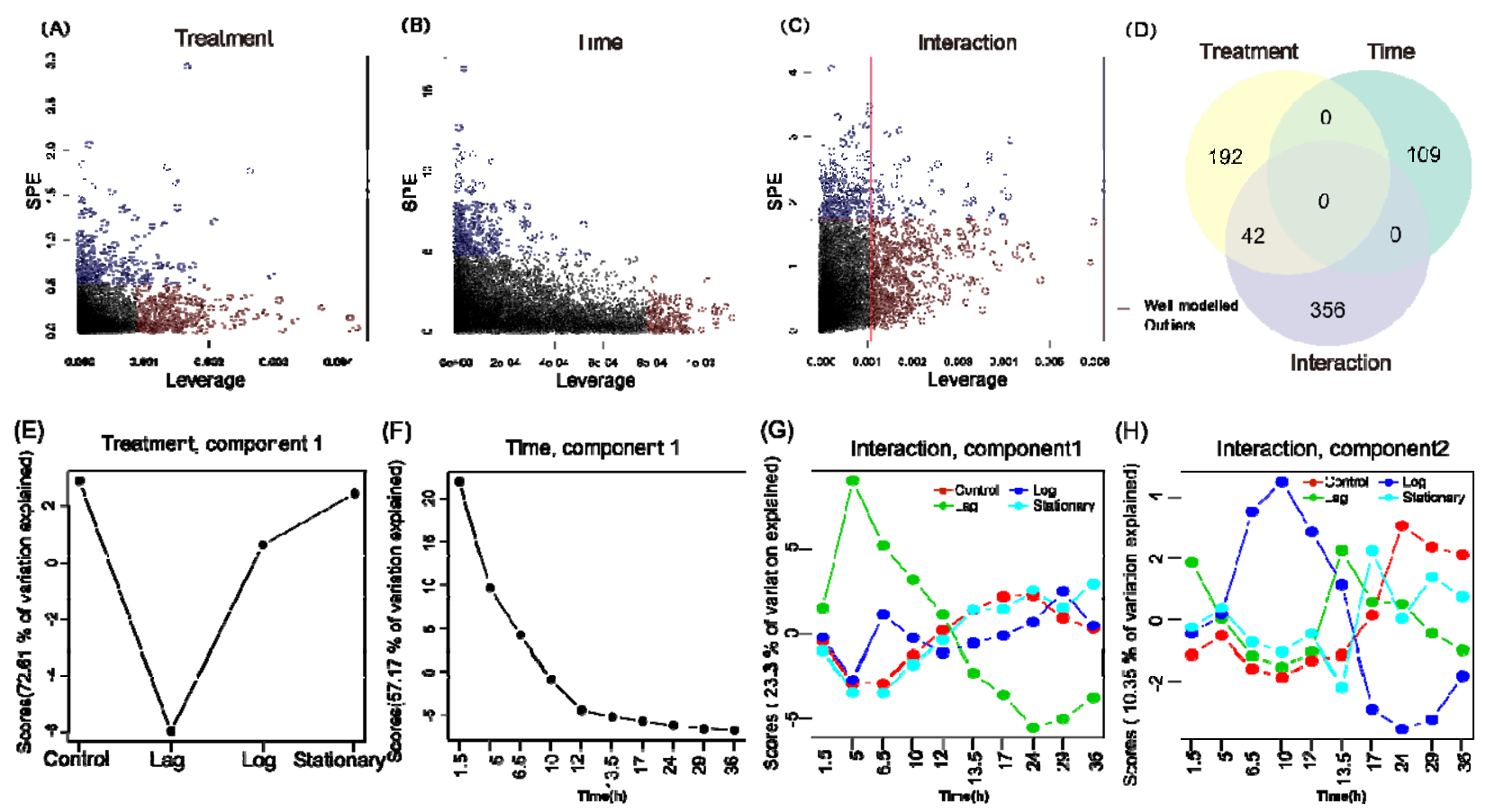

Figure.4 A time-series analysis using ANOVA - Simultaneous Component Analysis (ASCA).

SPE and leverage statistics of the protein groups in "Treatment" (A), "Time" (B) and "Interaction" (C) by the ANOVA-Simultaneous Component Analysis (ASCA). Squared prediction error (SPE) and leverage cut-off values were indicated by horizontal and vertical lines, respectively. In ASCA analysis, most relevant (well modeled, pink filled area) protein groups in the ASCA-model will be those showing high leverage and low SPE. (D) Venn diagram showed, in total, 699 well modeled protein groups were identified, of which 234 in 'Treatment', 109 in 'Time' and 398 in 'Interaction', respectively. Additionally, 42 protein groups were identified in 
'Treatment' and 'Interaction'. Score profiles of component 1 of 'Treatment' (E) and 'Time' (F). Score profiles of the first two components of sub model 'Interaction' (Treatment versus Time) (G-H).

\section{Taxonomic source and functional profiles of altered proteins were distinct when stimulated at different growth phases}

In ASCA analysis, the pattern of "Treatment" represents effect only due to metformin in all the groups. Therefore, to analyze the overall difference of taxon-specific functional responses of the gut microbiome stimulated at different growth phases, we selected well-modeled protein groups associated with 'Treatment' (with a high leverage and low SPE, Figure 4A) for detailed analysis. First, 234 well-modeled protein groups associated with 'Treatment' were identified (Supplementary Table S3). Then, hierarchical clustering and heatmap analysis revealed two different protein clusters (Figure 5A). Comparison of the expression profile of all protein groups in each cluster were shown in Figure 5B and E. In cluster I, the LFQ intensities of protein groups were lower in the Lag group as compared with the other treatment groups and the control (Figure 5B). In contrast, cluster II protein groups were higher in the Lag group (Figure 5E).

We performed functional and taxonomic enrichment analysis of the protein groups of clusters I and II using the "Enrichment analysis" app from iMetaLab (https://shiny.imetalab.ca/). To identify the taxonomic source of these proteins, the representative protein for each protein group in the dataset were submitted to the app, and enriched taxa with a $p$-value cut off of 0.05 were obtained (Figure 5C and F). Significantly reduced proteins from the Lag group were enriched in order Clostridiales and family Fusobacterium, species Subdoligranulum variabile, [Eubacterium] rectale, Fusobacterium mortiferum, Clostridium sp.M62/1 and Blautia wexlerae; while the increased proteins were enriched in genus Bacteroides and species Lachnospiraceae bacterium and Fusobacterium perfoetens. Interestingly, as shown in Figure S3, metformin added in the lag phase resulted in a sustained increase in the abundance of the genus Bacteroides after 10 hours of stimulation, which was different from the rest of treatment groups. 
We then performed functional enrichment analysis based on the database of clusters of orthologous groups (COG). All the significantly altered COGs and COG categories with a $p$ value cut-off of 0.05 were shown in Figure 5D and Supplementary Table S6. At the COG category level, in the Lag group, energy production and conversion, lipid transport and metabolism, translation, ribosomal structure and biogenesis were significantly decreased, no COG category was significantly enriched by the increased proteins (Figure 5D and G). However, we identified 47 COGs from 14 COG categories that were significantly increased (Supplementary Table S6) in the Lag group. Functions related to the carbohydrate transport and metabolism (7 COGs), Translation, ribosomal structure and biogenesis (7 COGs) were among the most significantly increased COGs.

We further analyzed the taxon-specific functional responses of the gut microbiome in different treatment groups at 6.5, 17 and $36 \mathrm{hr}$ (Supplementary Figures S4-S6). The results revealed that the gut microbiome responded differently to metformin added at different growth phases in each time point. For example, significantly altered protein groups at $36 \mathrm{hr}$ were identified by one-way ANOVA with Fisher's LSD test (FDR-adjusted p<0.05; Supplementary Table S4). Then, hierarchical clustering revealed five different protein clusters with $>10$ proteins (Figure S4A and B). Taxon and functional enrichment analysis were performed with the protein groups in each cluster (Figure S4C-D). To further analyze the taxon-specific functional difference in the three groups after the same number of hours of stimulation, we selected metaproteomic data after 12 and $24 \mathrm{hr}$ of stimulation for taxon-specific functional analysis, as show in Supplementary Figure S7 and S8, respectively. The significantly upregulated and downregulated protein groups in treatment groups compared to controls were identified by $t$-test (FDR-adjusted $\mathrm{p}<0.05$; Supplementary Table S5). Venn diagram indicates that after 12 or $24 \mathrm{hr}$ of stimulation, the most of significantly altered protein groups in the different treatment group were unique. The metaproteomic results revealed that the gut microbiome responded differently to metformin added at different growth phases, even at the same time after stimulation. The uniquely altered proteins of different treatment group were from different taxonomy and function. 
bioRxiv preprint doi: https://doi.org/10.1101/2020.02.05.936500; this version posted February 6, 2020. The copyright holder for this preprint (which was not certified by peer review) is the author/funder, who has granted bioRxiv a license to display the preprint in perpetuity. It is made available under aCC-BY-NC-ND 4.0 International license. 
bioRxiv preprint doi: https://doi.org/10.1101/2020.02.05.936500; this version posted February 6, 2020. The copyright holder for this preprint (which was not certified by peer review) is the author/funder, who has granted bioRxiv a license to display the preprint in perpetuity. It is made available under aCC-BY-NC-ND 4.0 International license.

(A)

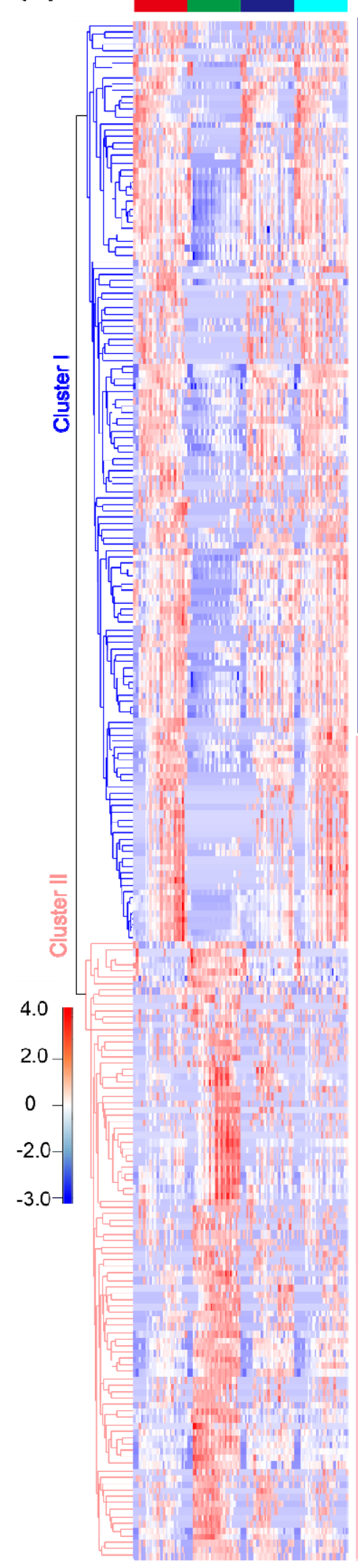

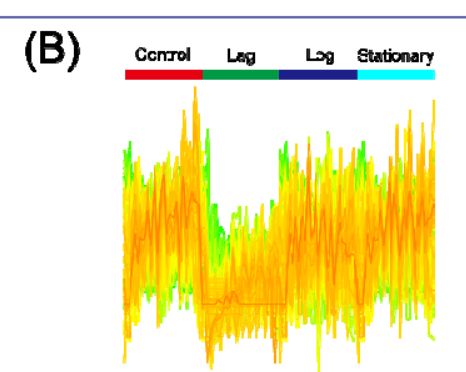

(D)
(C)
$-\log _{10}(\mathrm{p}$ value $)$

Subdolgranuhum variabile_spocies

[Eubecieriumprectaie_spocies

Podoviridae_farrily

Fusobacterium mortiferum_spscies

Clostridium sp.M62/_spscics

Fusobacteriaccec_farrily

Ruminococcaccac_farrily

Biautia vroxlorac_spocios |

Phascolarctobacteriurr_gonus |

Costridiales_order

[Ruminococcue]gnavus_species]

Fusobacteriurr_genus

Bacteroides thetaiotaomicron_spscres

Anaerotruncus sp. CAG:39C_spscies |:

Coprococcus_genus :

J 510,52025

Nurrer of protein groups

COG category level

$$
-\log _{10}(\rho \text { value) }
$$

$\begin{array}{llllll}0.0 & 0.2 & 0.4 & 0.8 & 0.5 & 1.0\end{array}$

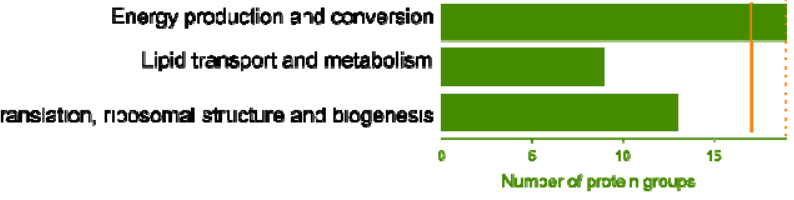

(E)

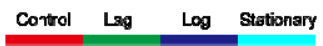

(F)

- $\log _{12}($ pvelue)

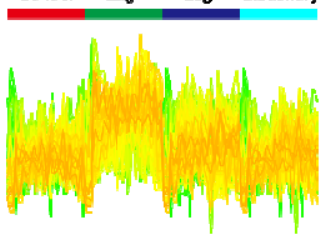

$\begin{array}{lllll}2 & 4 & 6 & 9 & 10\end{array}$

Bacteroides_genus

Lactinospiraceae bacierium species

Colfinselia gernus

Fusobacterium pertotens_species

Fusobacteriaçae_famity

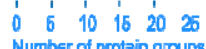

(G)

COG level

Number of protain groups

Prolein distantly rataled ic bacterial ferritins COG2406 FCF 1-iype ATP synthase, glphe subunit COG0050 Outer membrane protein W COG3047 MakateAsctate dehyodrogenase COG0039 Glutaminase COG2066

ABC-type dignepeptide transport systam, partplasmir component COG4166 Peraxiredoxin COG2077 Merhylmakonyi-COA mutase, N-terminal domarn/subunt COG1884 Enoyf-pacyl-camier-protein] reductase (NADH) COG0623 Nouraminiciass (sialidase) COG4409 Amincopotidaso C COC3579 Phosphohenose isomeras c000270

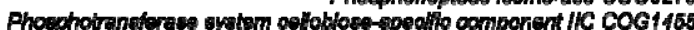
NDPH depandent 2,4 dienoy Cal reductase, atur reductase coco448

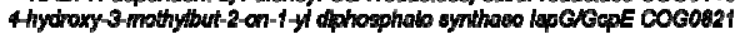

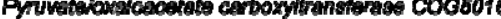
Othydroardede dehyctogenses 0000187 Molocutar chaperons topA HSP20 famlly COC0074 Trandation elengation factor CF,TU, a CTPase COC0050 Prrmale Co01013

Deoxybose-phosphate eldolase CO0027 CTPase involved in cell partioning and DNA nepeir Coc0030

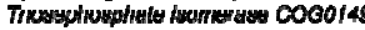
Enalase cocords 3-phosphoplycerate thase cocot20

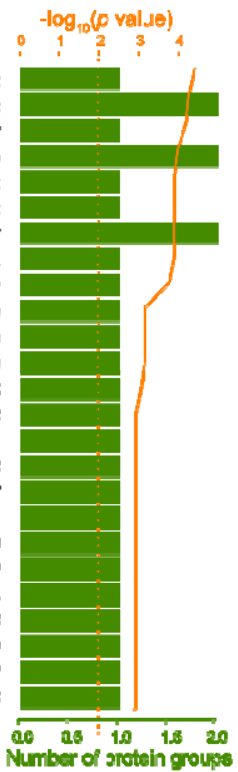


Figure.5 Metaproteomics revealed alterations of metaproteomic profiles when metformin added at different growth phases. (A) Heatmap of well-modeled protein groups associated with 'Treatment' by ASCA analysis. Protein groups with high leverage exhibit and low squared prediction error value are well-modeled. Heatmap shows the LFQ intensity of altered protein groups for each sample, 2 clusters (painted in blue and pink colors) were divided for further analyzed. (B, E) Alterations of protein groups in different groups in each cluster. $(\mathrm{C}, \mathrm{F})$ Alterations of taxonomic composition in each cluster. All the significantly taxonomy with a pvalue cut off of 0.05 are shown. (D) Alterations of functional composition (COG categories) in cluster I. (G) Top 25 most significant altered COGs in cluster II. No significant COG category was enrichment in cluster II. The significantly altered COG categories and COGs with a p-value cut off of 0.05. Bar charts showed the total numbers of protein groups corresponding to each taxonomy or function (FDR-adjusted $\mathrm{P}<0.05)$.

\section{Discussion}

In this study, we evaluated whether adding drug treatment at different growth phase of in vitro human gut microbiome would have different impacts on the functions of the microbiome. Usually, in vitro bacterial growth involves four phases: lag phase, log (exponential) phase, stationary phase, and death (decline) phase ${ }^{22,23}$. Bacteria adapt to the growth conditions in lag phase, which is the period the individual bacteria are maturing and not yet able to divide. The log phase is the period characterized by a logistic growth of bacterial biomass. The stationary phase results from a situation in which growth rate and death rate are equal, which is often due to a growth-limiting factor such as the depletion of an essential nutrient, and/or the formation of an inhibitory product such as an organic acid ${ }^{24}$. The death phase could be due to lack of nutrients and/or other injurious conditions ${ }^{22,23}$.

In this study, we observed that the growth curve of the microbiome is similar to the growth of bacterial pure cultures $^{25}$. We then performed three different treatments by adding metformin at the Lag, Log and stationary phases. We showed that the growth curves and metaproteomic 
profiles between treatment groups are significantly different. In agreement with previous reports $^{26,27}$, we also found that metformin has antibacterial action on in vitro gut microbiome (Figure 2A), especially after 24 hours of treatment. When subjected to nutrient limiting conditions, the bacteria become sensitive to metformin ${ }^{28}$. Stimulation at the log phase resulted in the strongest antibacterial action. It may well be that the antibacterial activity of metformin is dependent on the bacterial growth. The early studies on the mechanism of action of antibiotics showed that their bactericidal activity depends on bacterial growth ${ }^{18,19}$, our study suggested similar response of the growing microbiota to metformin. Previous study indicated that metformin could significantly protect animals from tested pathogenic bacteria and it has potential as a noteworthy antibacterial agent to be used in human ${ }^{27}$. Our research indicated that bacterial growth should be considered in future studies of the antibacterial mechanism and activity of metformin.

We showed that the metaproteomic profiles varied over different stimulation time points, with the earlier stimulation leading to the greatest effects on the gut microbiome. During lag phase, bacteria adapt to the growth conditions, and synthesis of ATP, DNA, RNA, enzymes and other molecules occurs ${ }^{22}$. Adding metformin at lag phase may significantly affect the protein function in these biological processes. We showed that metformin added at lag phase significantly decreased functions of energy production and conversion, lipid transport and metabolism, translation, ribosomal structure and biogenesis, which is in agreement with several previous studies $^{5,26,28}$. And metformin treatment also significantly increased the function about defense mechanisms, cell wall/membrane/envelope biogenesis. Most of these functions are closely related to the biological processes of bacteria in the lag phase $\mathrm{e}^{22}$, which may explain why the metformin added at the lag phase resulted in the strongest metaproteomic response.

Several studies showed that metformin can alter gut microbiota composition ${ }^{5,26,29,30}$, which also have been observed in our study. As with previous studies, our results indicated metformin causes a significantly increased abundance of the genus Bacteroide ${ }^{29,31,32}$, which was different from the rest of treatment groups. Metformin treatment at lag phase significantly increased defense mechanisms and cell wall/membrane/envelope biogenesis functions in the genus Bacteroides ${ }^{33,34}$. The species of the genus Bacteroides have complex systems to sense and adapt to the growth environment, and multiple pump systems to expel toxic substances, they 
have the most antibiotic resistance mechanisms and the highest resistance rates of all anaerobic bacteria $^{33,34}$. Therefore, our study may explain why metformin added at lag growth phase can increase the abundance of the genus Bacteroides.

In summary, our results suggest that drugs can have different effects depending on the growth phase of the microbiome and that in vitro systems are useful to assess these effects. The effects of drugs on the microbiome included changes in microbial biomass, functional profiles and their taxonomic contributions. Our work revealed that metformin added during the earliest growth phase had the strongest functional/compositional effect on the microbiome. However, the addition of metformin at log phase led to the greatest decline of bacterial growth. It could also mean that the effects of metformin in vivo might be affected by the phase of the microbiome. This in vitro approach would also be useful to identify compounds that have effects in different growth phases providing alternative strategies to target the microbiome.

\section{Methods}

\section{In vitro gut microbiome culturing and metformin treatments}

Here, we used a 96-deep well plate-based culturing model (MiPro) reported in our previous studies $^{26}$ for in vitro gut microbiome culturing. The medium composition for MiPro was based on our previously suggested medium composition ${ }^{35}$, which comprises: $2.0 \square \mathrm{g} \square \mathrm{L}^{-1}$ peptone water, $2.0 \square \mathrm{g} \square \mathrm{L}^{-1}$ yeast extract, $0.5 \square \mathrm{g} \square \mathrm{L}^{-1} \mathrm{~L}$-cysteine hydrochloride, $2 \square \mathrm{mL} \square \mathrm{L}^{-1}$ Tween 80 , $5 \square \mathrm{mg} \square \mathrm{L}^{-1}$ hemin, $10 \square \mu \mathrm{L} \square \mathrm{L}^{-1}$ vitamin $\mathrm{K} 1,1.0 \square \mathrm{g} \square \mathrm{L}^{-1} \mathrm{NaCl}, 0.4 \square \mathrm{g} \square \mathrm{L}^{-1} \mathrm{~K}_{2} \mathrm{HPO}_{4}, 0.4 \square \mathrm{g} \square \mathrm{L}^{-}$

${ }^{1} \mathrm{KH}_{2} \mathrm{PO}_{4}, 0.1 \square \mathrm{g} \square \mathrm{L}^{-1} \mathrm{MgSO}_{4} \cdot 7 \mathrm{H}_{2} \mathrm{O}, 0.1 \square \mathrm{g} \square \mathrm{L}^{-1} \mathrm{CaCl}_{2} \cdot 2 \mathrm{H}_{2} \mathrm{O}, 4.0 \square \mathrm{g} \square \mathrm{L}^{-1} \mathrm{NaHCO}_{3}, 4.0 \square \mathrm{g} \square \mathrm{L}^{-}$

${ }^{1}$ porcine gastric mucin (cat\# M1778, Sigma-Aldrich), $0.25 \mathrm{~g} \mathrm{~L}^{-1}$ sodium cholate and $0.25 \mathrm{~g} \mathrm{~L}^{-1}$ sodium chenodeoxycholate.

In this study, a stool sample was obtained from a healthy volunteer (age 31 years; male). The participant signed informed consent and the sampling protocol was approved by the Ottawa Health Science Network Research Ethics Board at the Ottawa Hospital (\#20160585-01H). Stool was collected in pre-reduced sterile PBS buffer, and was immediately homogenized and filtered 
with sterile gauze in an anaerobic workstation $\left(5 \% \mathrm{H}_{2}, 5 \% \mathrm{CO}_{2}\right.$, and $90 \% \mathrm{~N}_{2}$ at $\left.37 \square{ }^{\circ} \mathrm{C}\right)$. The filtrate was aliquoted in $1.5 \mathrm{ml}$ Eppendorf tubes and stored at $-80{ }^{\circ} \mathrm{C}$.

When culturing, the frozen inoculums were taken out and placed in a $37^{\circ} \mathrm{C}$ water bath for 10 minutes, immediately inoculated at a concentration of $2 \%(w / v)$ into a 96 -well deep well plate containing $1 \mathrm{ml}$ culture medium. Concentration of metformin (metformin hydrochloride) was determined based on the assumption that maximal oral dosage of the drug distributed in $200 \mathrm{~g}$ average weight of the colon contents, i.e. $5 \mu$ f filter-sterilized water solution of metformin was added into each of the designated wells to achieve a final drug concentration of $7.6 \mathrm{mM}{ }^{26}$. All samples are cultured for optical density and metaproteomics analyses in technical triplicates at each different time point.

\section{Growth curve fitting with logistic equation}

A biobanked human gut microbiome sample were inoculated into the MiPro medium without metformin for culturing, triplicates of wells were collected at 0 (immediately after inoculation), $1.5,3,5,6.5,8,10,12,13.5,32$, and $48 \square \mathrm{hr}$, respectively. Optical density of each sample was measured at $595 \mathrm{~nm}\left(\mathrm{OD}_{595}\right)$, as a proxy of microbial biomass. Briefly, $100 \square \mu l$ aliquots were removed from each well, centrifuged at $16,000 \square \times g$ and the supernatant was used as the medium blank for the $\mathrm{OD}_{595}$ measurement. The growth curve is fitted with a logistic growth model by the $\mathrm{R}$ package Growthcurver based on the $\mathrm{OD}_{595}$ value over 48 hours ${ }^{36}$. The logistic equation is a common standard form in ecology and evolution ${ }^{37,38}$, whose parameters (the growth rate, the initial population size, and the carrying capacity) provide meaningful population-level information with straight-forward biological interpretation. For comparing the effect of stimulation times on microbiome response to metformin, we determinate metformin was added at the $0 \mathrm{hr}$ of the lag phase, $5 \mathrm{hr}$ of the log phase and $12 \mathrm{hr}$ of the stationary phase, respectively, according to the growth curve. Then, following each metformin treatment, the samples in each group were collected at collected at 0 (immediately after inoculation), 1.5, 3, 5, 6.5, 8, 10, 12, $13.5,15,17,20,24,29$, and $36 \square \mathrm{hr}$ for microbial biomass analysis, respectively, as shown in Supplementary Table S2. 


\section{Metaproteomic sample processing and LC-MS/MS analysis}

Following each metformin treatment, the samples were collected at $0,1.5,5,6.5,10,12,13.5$, 17, 24, 29, and 36 $\square \mathrm{hr}$ for metaproteomic analyses, respectively, as shown in Supplementary Table S2. The collected samples were washed twice by PBS with centrifugation at 14,000 $\square \mathrm{g}$, $4 \square{ }^{\circ} \mathrm{C}$ for $20 \square \mathrm{min}$. Then this pellet fraction was then harvested for further metaproteomics analysis. Protein extraction and trypsin digestion were performed according to our previously published procedure ${ }^{39}$. Briefly, the microbial cell pellets were lysed with protein lysis buffer containing $4 \%$ (w/v) sodium dodecyl sulfate (SDS), 8$\square \mathrm{M}$ urea in $50 \square \mathrm{mM}$ Tris- $\mathrm{HCl}$ buffer (pH 8.0), Roche PhosSTOPTM and Roche cOmplete ${ }^{\mathrm{TM}}$ Mini tablets. Lysates were sonicated with a Q800R3 sonicator (QSonica) for $5 \mathrm{~min}$ at $4{ }^{\circ} \mathrm{C}$ (60 secs on/off, 70\% amplitude). Then Removing cell debris through high-speed centrifugation at $16,000 \square \mathrm{g}, 4 \square{ }^{\circ} \mathrm{C}$ for $10 \square \mathrm{min}$. The resulting supernatant was precipitated using acidified acetone/ethanol buffer at $-20 \square{ }^{\circ} \mathrm{C}$ overnight.

After centrifugation at $16,000 \square \mathrm{g}$ for $20 \square \mathrm{min}$ at $4 \square{ }^{\circ} \mathrm{C}$, the pelleted proteins were washed three times with ice-cold acetone, then dissolved in $6 \square \mathrm{M}$ urea in $50 \square \mathrm{mM}$ ammonium bicarbonate $(\mathrm{pH}$ $=8)$. Then, $50 \mu \mathrm{g}$ of protein in each sample was reduced and alkylated with $10 \square \mathrm{mM}$ dithiothreitol and $20 \square \mathrm{mM}$ iodoacetamide, respectively. One microgram of trypsin (Worthington Biochemical Corp., Lakewood, NJ) was then added and shaken for digestion at $37 \square{ }^{\circ} \mathrm{C}$ overnight. Protein concentrations were determined by DC (detergent compatible) protein assay.

The tryptic peptides were desalted with a 10- $\mu \mathrm{m}$ C18 column and desalted tryptic peptides corresponding to $0.2 \square \mu \mathrm{g}$ of protein were loaded for LC-MS/MS analysis with an Eskigent 425 nanoLC and run on a Q Exactive HF-X mass spectrometer (ThermoFisher Scientific Inc.). Peptides were separated with an analytical column (75 $\square$ m m i.d. $\square \times \square 15 \square \mathrm{cm})$ packed with reverse phase beads (1.9 $\square \mu \mathrm{m} ; 120-\AA ̊$ pore size; Dr. Maisch GmbH, Ammerbuch, Germany). Peptide separation was performed using a $90 \square \min$ gradient from 5 to $35 \%$ (v/v) acetonitrile at a flow rate of $300 \mathrm{nl} / \mathrm{min}$. The instrument parameters included a full MS scan from 300 to $1800 \square \mathrm{m} / \mathrm{z}$, followed by data-dependent MS/MS scan of the 16 most intense ions, a dynamic exclusion repeat count of two, and repeat exclusion duration of $30 \square \mathrm{s}$. All samples were run on LC-MS/MS in a random order. In addition, nine samples were randomly selected for MS QC runs. 


\section{Metaproteomics data processing}

The MS data was processed with MetaPro-IQ bioinformatics workflow ${ }^{39}$ by the MetaLab software (version 1.0) ${ }^{21}$ for peptide/protein identification and quantification. Database construction were based on an iterative database search strategy using gut microbial gene catalogs (from http://meta.genomics.cn/) and a spectral clustering strategy. The identified protein lists were generated with a target-decoy strategy based on a FDR cut-off of 0.01. Label-free quantification (LFQ) intensity of proteins across all samples were obtained with the MaxLFQ algorithm $^{17}$. Taxonomic and functional enrichment analysis was performed using the enrichment module on iMetalab.ca ${ }^{40}$ through inputting the list of altered proteins groups. The database (version 2014) of clusters of orthologous groups (COG) of proteins was used for functional annotation $^{39}$.

\section{Statistical and multivariate data analysis}

In order to compare the difference among the different groups in microbial biomass and protein groups at each time point, the data at the missing time points were imputed using the $\mathrm{R}$ package imputeTS ${ }^{41}$, which specializes on univariate time series imputation. The quantified protein groups were filtered with the criteria that the protein should be present in $\geq 25 \%$ of the samples (Q25). And one sample were screened out due to ineligible sample quality $(<1 / 2$ average peptide identification). Then LFQ protein group intensities of the filtered file was log2-transformed and normalized through quotient transformation (x/mean) using the MetaboAnalyst (http://www.metaboanalyst.ca/) ${ }^{42}$, as shown in Supplementary Table S7.

To compare the difference in growth curve and metaproteomic profiles between different treatment times, t-test was performed at the time points for 1.5-5 hr (control vs. Lag only), and one-way ANOVA was performed at the time points for 6.5-36 hr. The metaproteomics data was time-course data, involving different treatments (added metformin at different growth phase as a different treatment) and sampling time points. For the time-series analysis, ANOVASimultaneous Component Analysis (ASCA) was used to identify major patterns with regard to the two given factors (Treatment and Time) and their interaction (Treatment versus Time). The algorithm first partitions the overall data variance $(\mathrm{X})$ into individual variances induced by each 
factor (Treatment and Time), as well as by the interactions (Treatment versus Time) ${ }^{43}$. The formula is shown below with $(\mathrm{E})$ indicates the residual Errors: $\mathrm{X}=$ Treatment + Time + Treatment versus Time + E. To test the significance of the effects associated with main effects, we performed a model validation, which is based on the Manly's unrestricted permutation of observation then calculate the permuted variation associated with each factor. In general, protein groups with high leverage exhibit and low squared prediction error (SPE) value in ASCA analysis, which means that significant model contributions are also well modeled protein groups $^{44}$. Cut off values for SPE and leverage were computed as described in the methods section taking $\alpha=0.05$. The t-test, ANOVA, ASCA analysis and Principal component analysis (PCA) was performed in MetaboAnalyst $4.0^{45}$. Hierarchical clustering analysis and heatmap plotting was performed with Perseus (version 1.6.10.0) ${ }^{46}$. Multivariate analysis of variance (MANOVA) was performed in MATLAB (The MathWorks Inc.).

\section{Acknowledgments}

This work was supported by the Government of Canada through Genome Canada and the Ontario Genomics Institute (OGI-156), the Natural Sciences and Engineering Research Council of Canada (NSERC, grant no. 210034), and the Ontario Ministry of Economic Development and Innovation (ORF-DIG-14405).

\section{Author contributions}

Z.H., L.L., H.L., and D.F. designed the study. D.F. supervised the study. Z.H, L.L., Z.N., and K.W. performed the experiments. Z.H, L.L., Z.N., X. Z. and K.C. performed data analysis. Z.H., L.L., and D.F. wrote the paper. H.L., X.Z., J.M., K.W., and Z.N. contributed to the editing and revision of the paper. All authors read and approved the final manuscript.

\section{Disclosure of Potential Conflicts of Interest}

No potential conflicts of interest were disclosed. 
1. Lam KN, Alexander M, Turnbaugh PJ. Precision medicine goes microscopic: engineering the microbiome to improve drug outcomes. Cell host \& microbe 2019; 26:22-34.

2. Cani PD, Delzenne NM. The gut microbiome as therapeutic target. Pharmacology \& therapeutics 2011; 130:202-12.

3. Imhann F, Vich Vila A, Bonder MJ, Lopez Manosalva AG, Koonen DP, Fu J, et al. The influence of proton pump inhibitors and other commonly used medication on the gut microbiota. Gut Microbes 2017; 8:351-8.

4. Pallav K, Dowd SE, Villafuerte J, Yang X, Kabbani T, Hansen J, et al. Effects of polysaccharopeptide from Trametes versicolor and amoxicillin on the gut microbiome of healthy volunteers: a randomized clinical trial. Gut microbes 2014; 5:458-67.

5. Wu H, Esteve E, Tremaroli V, Khan MT, Caesar R, Mannerås-Holm L, et al. Metformin alters the gut microbiome of individuals with treatment-naive type 2 diabetes, contributing to the therapeutic effects of the drug. Nature medicine 2017; 23:850.

6. Walsh J, Griffin BT, Clarke G, Hyland NP. Drug-gut microbiota interactions: implications for neuropharmacology. British journal of pharmacology 2018; 175:4415-29.

7. Jalili-Firoozinezhad S, Gazzaniga FS, Calamari EL, Camacho DM, Fadel CW, Bein A, et al. A complex human gut microbiome cultured in an anaerobic intestine-on-a-chip. Nature Biomedical Engineering 2019; 3:520-31.

8. Shah P, Fritz JV, Glaab E, Desai MS, Greenhalgh K, Frachet A, et al. A microfluidics-based in vitro model of the gastrointestinal human-microbe interface. Nature Communications 2016; 7:11535.

9. Macedo MH, Araujo F, Martinez E, Barrias C, Sarmento B. iPSC-Derived enterocyte-like cells for drug absorption and metabolism studies. Trends in molecular medicine 2018; 24:696-708.

10. Wu H, Esteve E, Tremaroli V, Khan MT, Caesar R, Mannerås-Holm L, et al. Metformin alters the gut microbiome of individuals with treatment-naive type 2 diabetes, contributing to the therapeutic effects of the drug. Nature Medicine 2017; 23:850.

11. Verhoeckx K, Cotter P, López-Expósito I, Kleiveland C, Lea T, Mackie A, et al. The impact of food bioactives on health: in vitro and ex vivo models. Springer, 2015.

12. Molly K, Woestyne MV, Smet ID, Verstraete W. Validation of the simulator of the human intestinal microbial ecosystem (SHIME) reactor using microorganism-associated activities. Microbial Ecology in Health and Disease 1994; 7:191-200.

13. Giuliani C, Marzorati M, Innocenti M, Vilchez-Vargas R, Vital M, Pieper DH, et al. Dietary supplement based on stilbenes: a focus on gut microbial metabolism by the in vitro simulator $\mathrm{M}$ SHIME ${ }^{\circledR}$. Food \& function 2016; 7:4564-75.

14. Kim HJ, Li H, Collins JJ, Ingber DE. Contributions of microbiome and mechanical deformation to intestinal bacterial overgrowth and inflammation in a human gut-on-a-chip. Proceedings of the National Academy of Sciences 2016; 113:E7-E15.

15. Li L, Abou-Samra E, Ning Z, Zhang X, Mayne J, Wang J, et al. An in vitro model maintaining taxonspecific functional activities of the gut microbiome. Nature communications 2019; 10:1-11. 
16. Maier L, Pruteanu M, Kuhn M, Zeller G, Telzerow A, Anderson EE, et al. Extensive impact of nonantibiotic drugs on human gut bacteria. Nature 2018; 555:623-8.

17. Wang $X$, Sun G, Feng T, Zhang J, Huang X, Wang T, et al. Sodium oligomannate therapeutically remodels gut microbiota and suppresses gut bacterial amino acids-shaped neuroinflammation to inhibit Alzheimer's disease progression. Cell research 2019; 29:787-803.

18. Hobby GL, Dawson MH. Effect of Rate of Growth of Bacteria on Action of Penicillin. Proceedings of the Society for Experimental Biology and Medicine 1944; 56:181-4.

19. Hobby GL, Meyer K, Chaffee E. Observations on the Mechanism of Action of Penicillin.

Proceedings of the Society for Experimental Biology and Medicine 1942; 50:281-5.

20. Tuomanen E, Cozens R, Tosch W, Zak O, Tomasz A. The rate of killing of Escherichia coli byßlactam antibiotics is strictly proportional to the rate of bacterial growth. Microbiology 1986; 132:1297304.

21. Cheng K, Ning Z, Zhang X, Li L, Liao B, Mayne J, et al. MetaLab: an automated pipeline for metaproteomic data analysis. Microbiome 2017; 5:157.

22. Widdel F. Theory and measurement of bacterial growth. Di dalam Grundpraktikum Mikrobiologie 2007; 4:1-11.

23. Funke BR, Case CL, Gerard J, Tortora G. Microbiology: an introduction. Benjamin/Cummings, 1998.

24. Bridges BA, Foster PL, Timms AR. Effect of endogenous carotenoids on "adaptive" mutation in Escherichia coli FC40. Mutation Research/Fundamental and Molecular Mechanisms of Mutagenesis 2001; 473:109-19.

25. Zwietering MH, Jongenburger I, Rombouts FM, van 't Riet K. Modeling of the Bacterial Growth Curve. Applied and Environmental Microbiology 1990; 56:1875-81.

26. Guo H-H, Feng C-L, Zhang W-X, Luo Z-G, Zhang H-J, Zhang T-T, et al. Liver-target nanotechnology facilitates berberine to ameliorate cardio-metabolic diseases. Nature Communications 2019; 10:1981.

27. Nasrin F. Study of anti-microbial and anti-oxidant potentiality of anti-diabetic drug metformin. International journal of pharmaceutics and drug analysis 2014; 3:220-4.

28. Liu X, Romero IL, Litchfield LM, Lengyel E, Locasale JW. Metformin targets central carbon metabolism and reveals mitochondrial requirements in human cancers. Cell metabolism 2016; 24:72839.

29. Zhang X, Zhao Y, Xu J, Xue Z, Zhang M, Pang X, et al. Modulation of gut microbiota by berberine and metformin during the treatment of high-fat diet-induced obesity in rats. Scientific reports 2015; 5:14405.

30. Forslund K, Hildebrand F, Nielsen T, Falony G, Le Chatelier E, Sunagawa S, et al. Disentangling type 2 diabetes and metformin treatment signatures in the human gut microbiota. Nature 2015; 528:262.

31. Lee H, Lee $\mathrm{Y}, \mathrm{Kim} J$, An J, Lee $\mathrm{S}$, Kong $\mathrm{H}$, et al. Modulation of the gut microbiota by metformin improves metabolic profiles in aged obese mice. Gut Microbes 2018; 9:155-65.

32. Bryrup T, Thomsen CW, Kern T, Allin KH, Brandslund I, Jørgensen NR, et al. Metformin-induced changes of the gut microbiota in healthy young men: results of a non-blinded, one-armed intervention study. Diabetologia 2019; 62:1024-35.

33. Wexler HM. Bacteroides: the good, the bad, and the nitty-gritty. Clinical microbiology reviews 2007; 20:593-621.

34. Soki J, Gal M, Brazier JS, Rotimi VO, Urban E, Nagy E, et al. Molecular investigation of genetic elements contributing to metronidazole resistance in Bacteroides strains. Journal of Antimicrobial Chemotherapy 2005; 57:212-20. 
35. Li L, Zhang X, Ning Z, Mayne J, Moore JI, Butcher J, et al. Evaluating in Vitro Culture Medium of Gut Microbiome with Orthogonal Experimental Design and a Metaproteomics Approach. J Proteome Res 2018; 17:154-63.

36. Sprouffske K, Wagner A. Growthcurver: an R package for obtaining interpretable metrics from microbial growth curves. BMC Bioinformatics 2016; 17:172.

37. Crow JF, Kimura M. An introduction to population genetics theory. An introduction to population genetics theory 1970 .

38. Rockwood LL. Introduction to population ecology. John Wiley \& Sons, 2015.

39. Zhang X, Ning Z, Mayne J, Moore JI, Li J, Butcher J, et al. MetaPro-IQ: a universal metaproteomic approach to studying human and mouse gut microbiota. Microbiome 2016; 4:31.

40. Liao B, Ning Z, Cheng K, Zhang X, Li L, Mayne J, et al. iMetaLab 1.0: a web platform for metaproteomics data analysis. Bioinformatics 2018; 34:3954-6.

41. Moritz S, Bartz-Beielstein T. imputeTS: time series missing value imputation in R. The R Journal 2017; 9:207-18.

42. Chong J, Soufan O, Li C, Caraus I, Li S, Bourque G, et al. MetaboAnalyst 4.0: towards more transparent and integrative metabolomics analysis. Nucleic acids research 2018; 46:W486-W94.

43. Smilde AK, Jansen JJ, Hoefsloot HC, Lamers R-JA, Van Der Greef J, Timmerman ME. ANOVAsimultaneous component analysis (ASCA): a new tool for analyzing designed metabolomics data. Bioinformatics 2005; 21:3043-8.

44. Nueda MJ, Conesa A, Westerhuis JA, Hoefsloot HC, Smilde AK, Talón M, et al. Discovering gene expression patterns in time course microarray experiments by ANOVA-SCA. Bioinformatics 2007; 23:1792-800.

45. Chong J, Wishart DS, Xia J. Using metaboanalyst 4.0 for comprehensive and integrative metabolomics data analysis. Current Protocols in Bioinformatics 2019; 68:e86.

46. Tyanova S, Temu T, Sinitcyn P, Carlson A, Hein MY, Geiger T, et al. The Perseus computational platform for comprehensive analysis of (prote)omics data. Nature Methods 2016; 13:731. 
(A) Hypothesis: Drug added at different growth phase

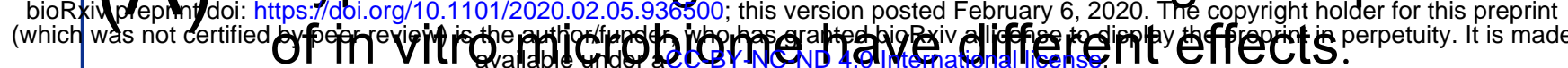
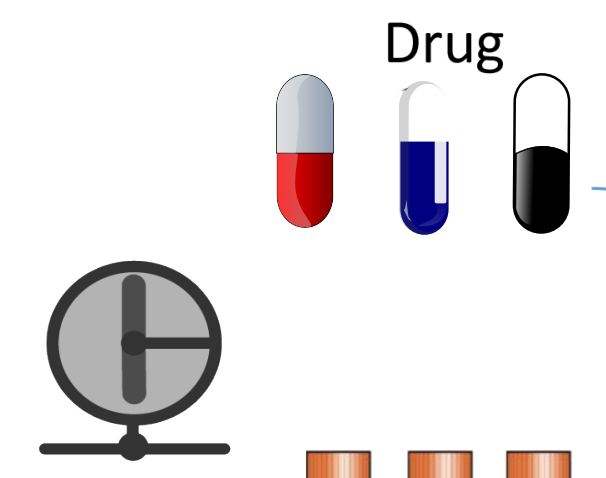

西 101.

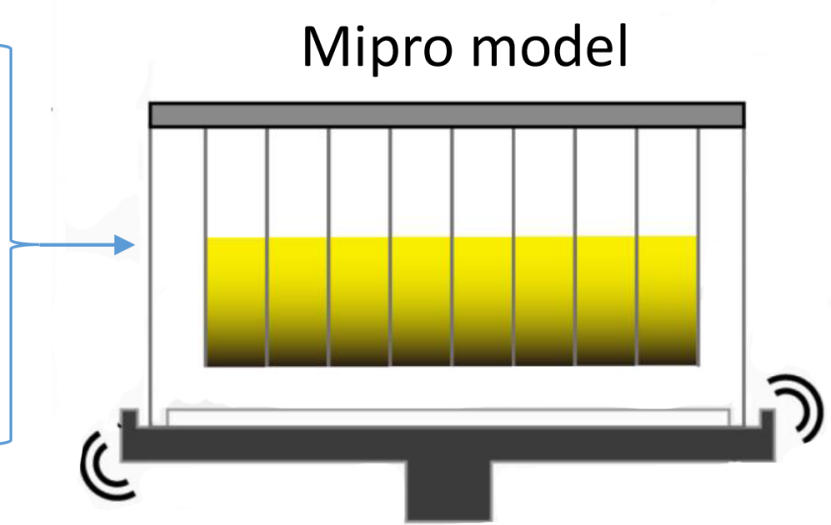

Gut microbiome

(B) Determining the growth curve and stimulation times

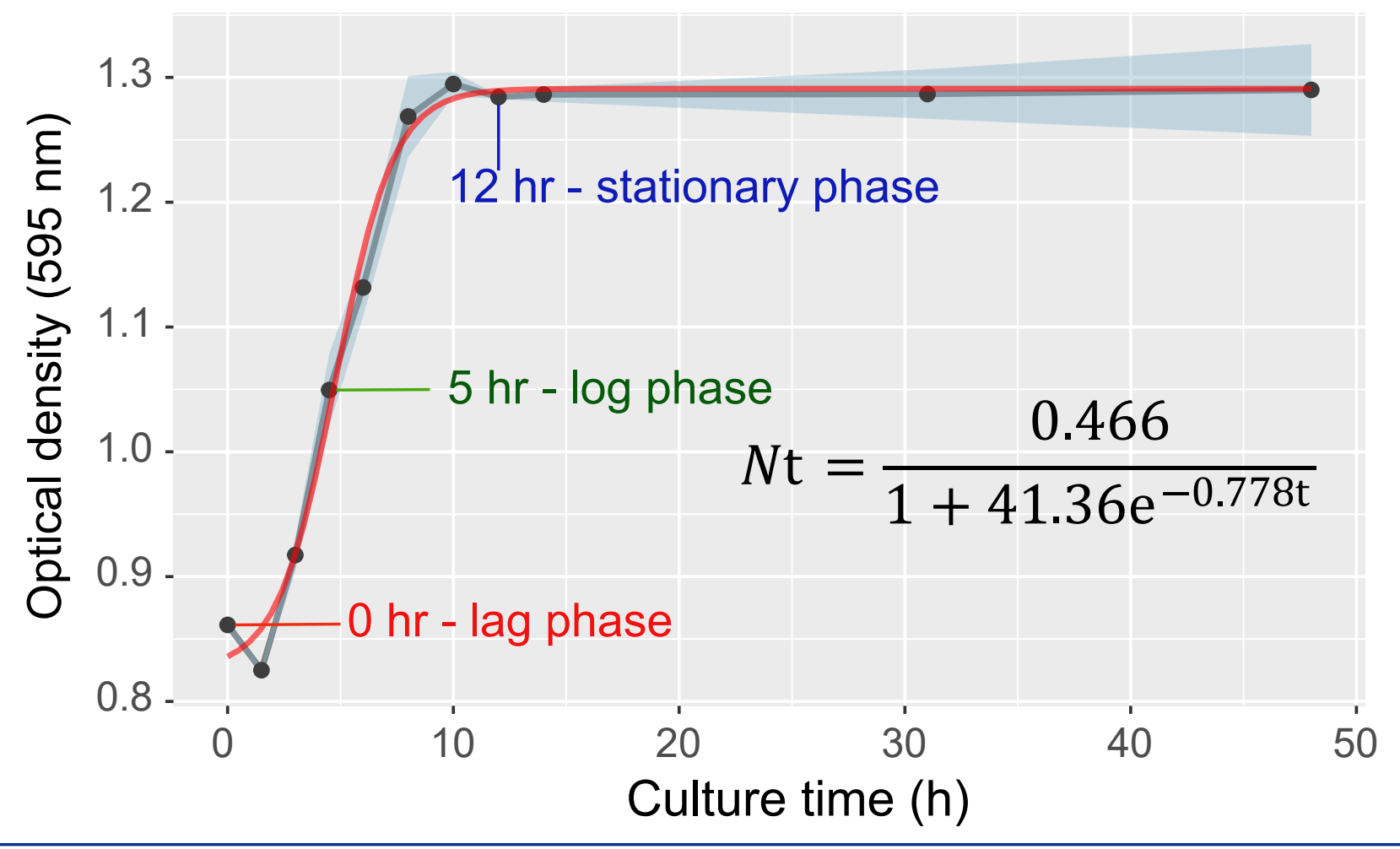

(C)
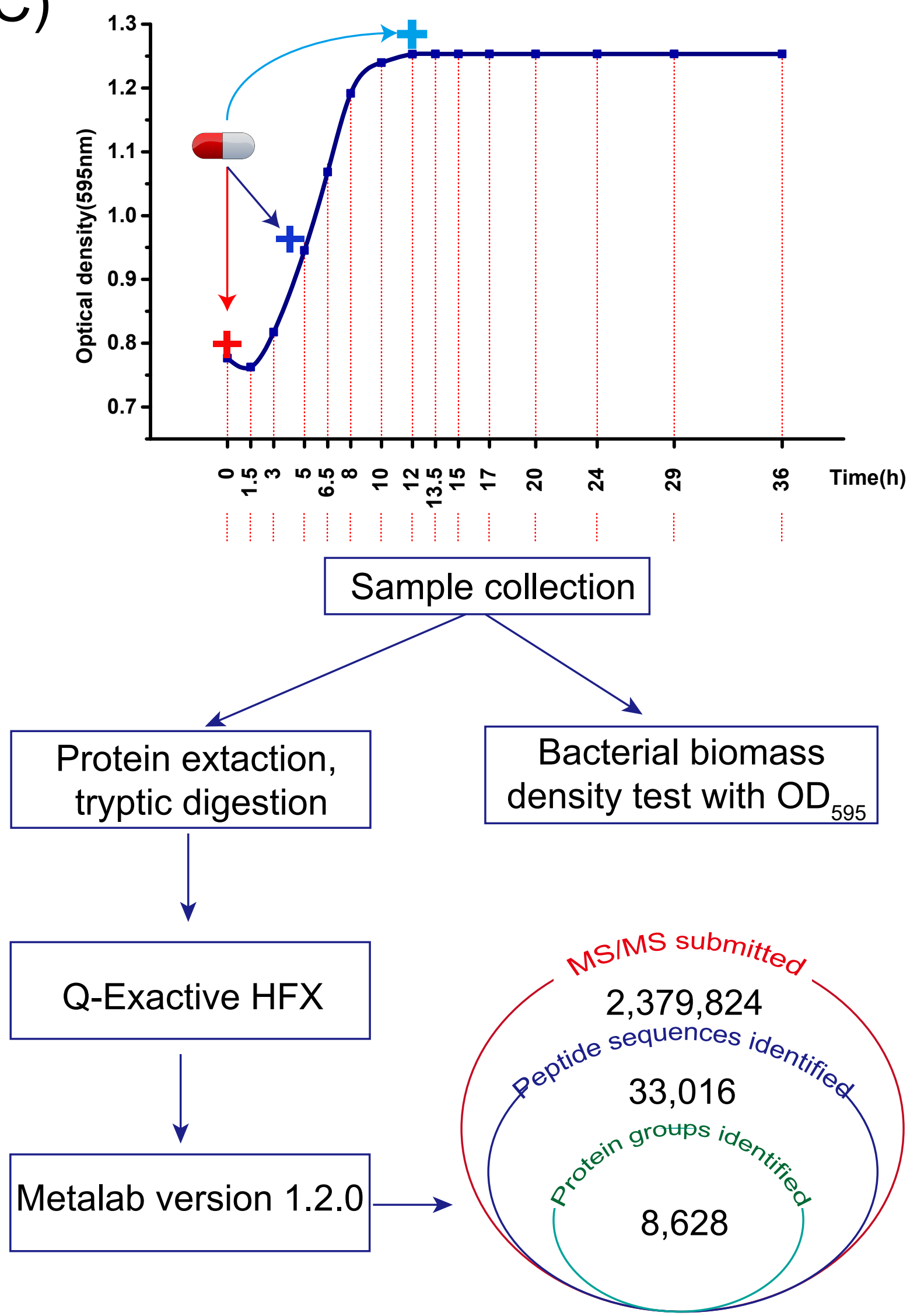


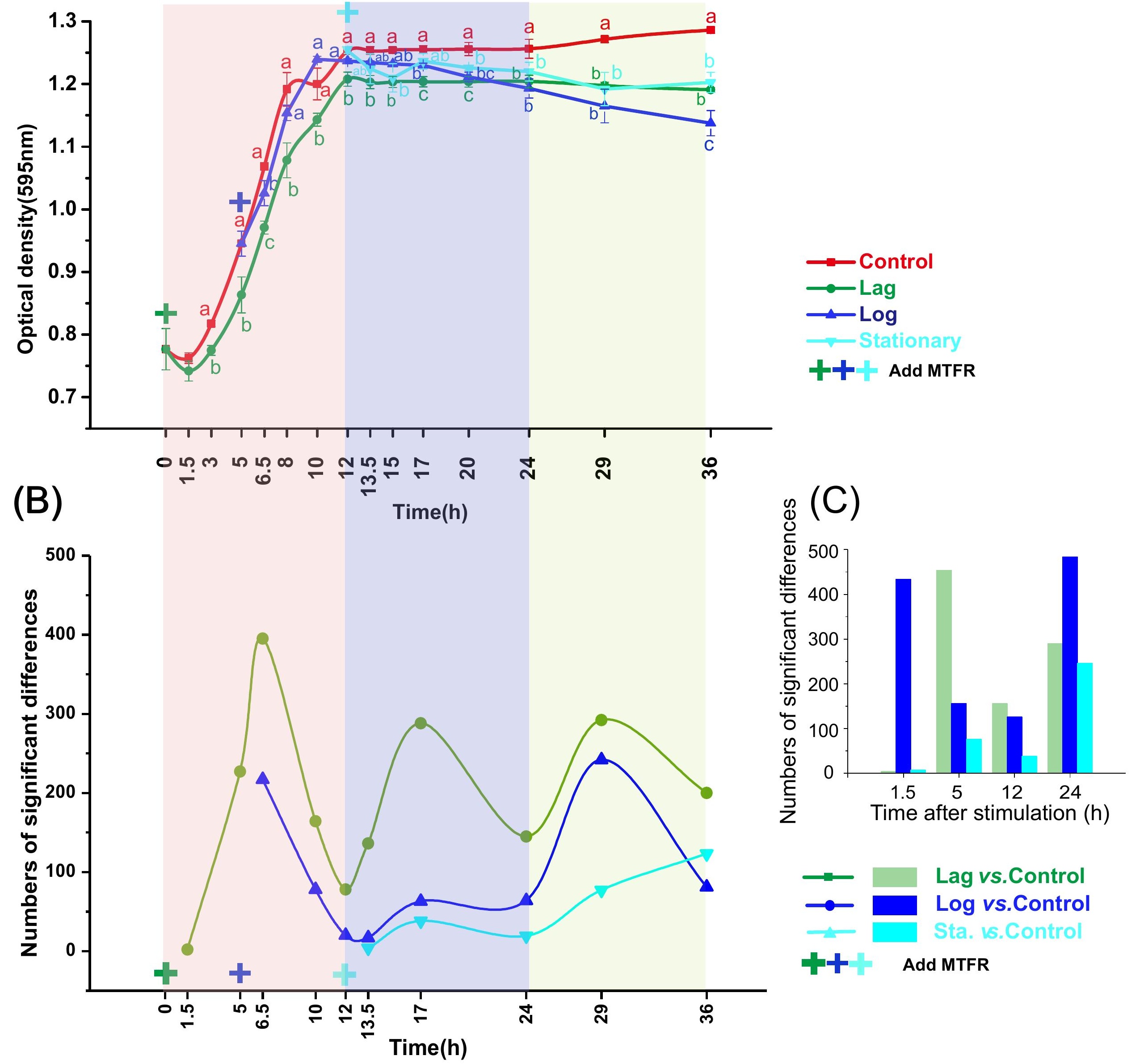




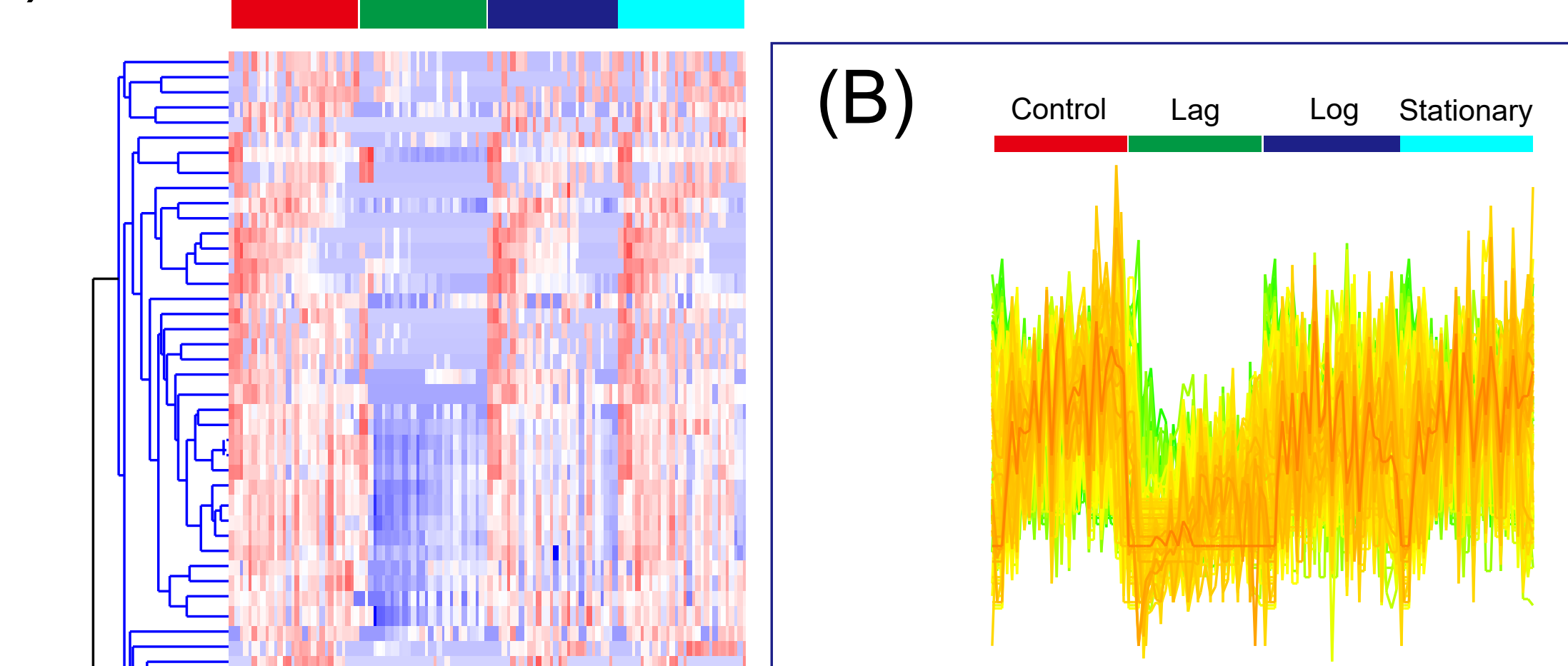

(C)

Subdoligranulum variabile_species

[Eubacterium]rectale_species

Podoviridae_family

Fusobacterium mortiferum_species

Clostridium sp.M62/1_species

Fusobacteriaceae_family

Ruminococcaceae_family

Blautia wexlerae_species

Phascolarctobacterium_genus

Clostridiales_order

[Ruminococcus]gnavus_species

Fusobacterium_genus

Bacteroides thetaiotaomicron_species

Anaerotruncus sp. CAG:390_species

Coprococcus_genus

(D)

$\begin{array}{llllll}0 & 5 & 10 & 15 & 20 & 25\end{array}$

Number of protein groups

COG category level

$-\log _{10}(p$ value $)$

Energy production and conversion

Lipid transport and metabolism

Translation, ribosomal structure and biogenesis

$\begin{array}{llllll}0.0 & 0.2 & 0.4 & 0.6 & 0.8 & 1.0\end{array}$

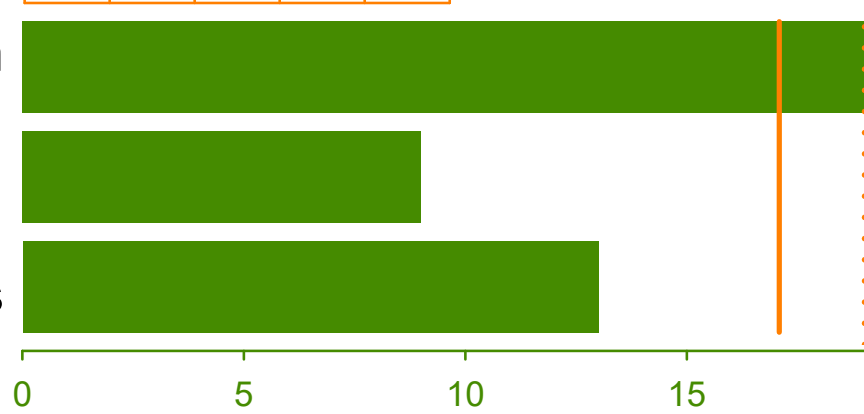

Number of protein groups

7f 6ruary 2020. The copyright holder for this preprint

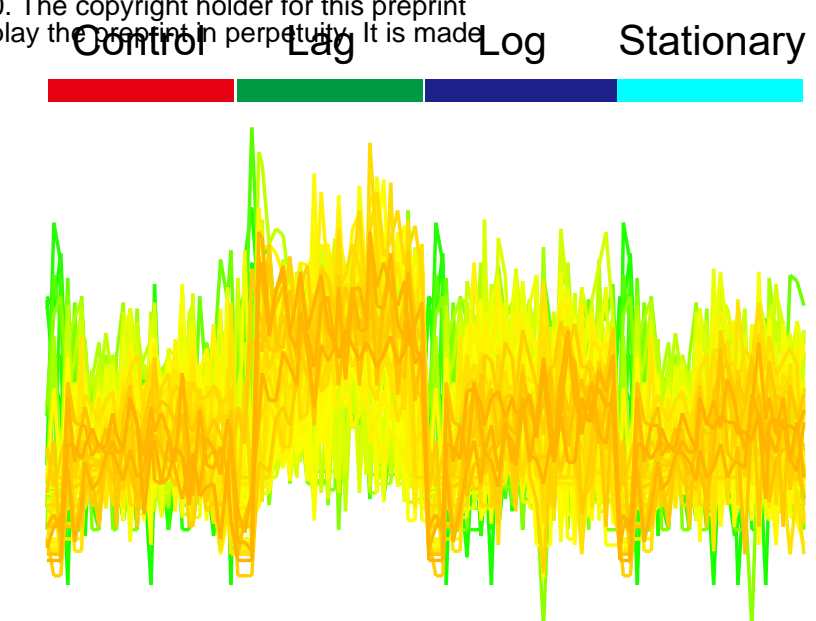

$(\mathrm{F})$

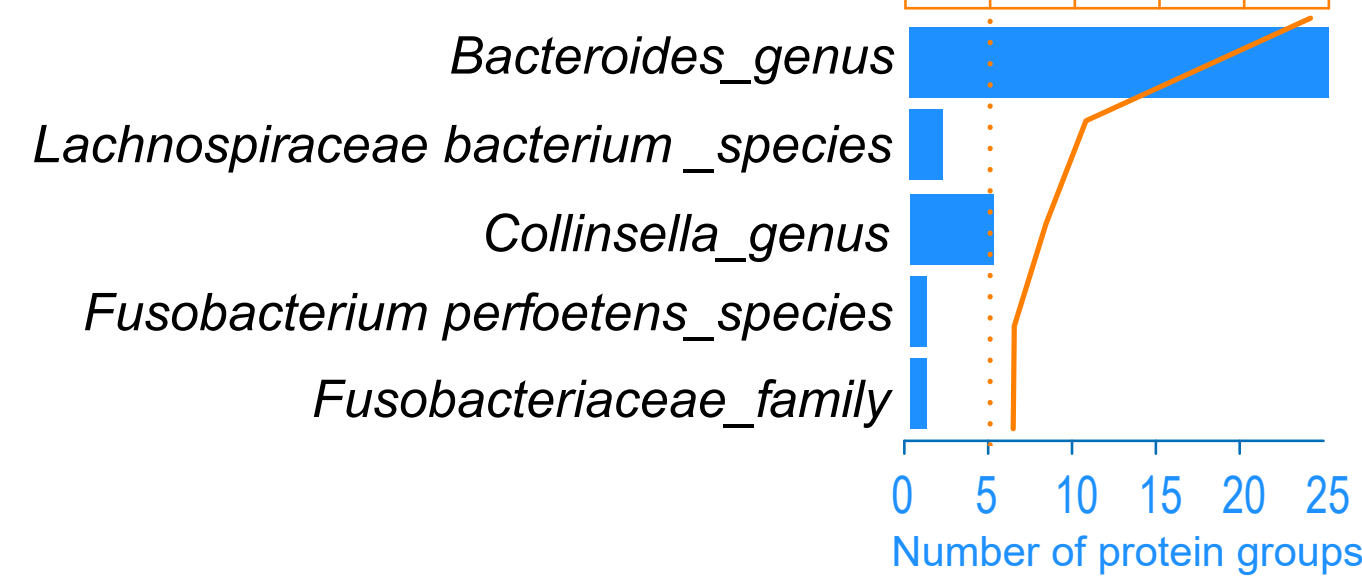

(G)

COG level

Protein distantly related to bacterial ferritins COG2406 FoF1-type ATP synthase, alpha subunit COG0056 Outer membrane protein W COG3047 Malate/lactate dehydrogenase COG0039 Glutaminase COG2066 ABC-type oligopeptide transport system, periplasmic component COG4166 Peroxiredoxin COG2077 Methylmalonyl-CoA mutase, N-terminal domain/subunit COG1884 Enoyl-[acyl-carrier-protein] reductase (NADH) COG0623 Neuraminidase (sialidase) COG4409 Aminopeptidase C COG3579 Phosphoheptose isomerase COG0279

Phosphotransferase system cellobiose-specific component IIC COG1455

NADPH-dependent 2,4-dienoyl-CoA reductase, sulfur reductase COG0446 4-hydroxy-3-methylbut-2-en-1-yl diphosphate synthase IspG/GcpE COG0821

Pyruvate/oxaloacetate carboxyltransferase COG5016 Dihydroorotate dehydrogenase COG0167

Molecular chaperone IbpA, HSP20 family COG0071 Translation elongation factor EF-Tu, a GTPase COG0050 Pyruvate COG1013

Deoxyribose-phosphate aldolase COG0274 GTPase involved in cell partioning and DNA repair COG0536 Triosephosphate isomerase COG0149 Enolase COG0148 3-phosphoglycerate kinase COG0126 $-\log _{10}(p$ value $)$

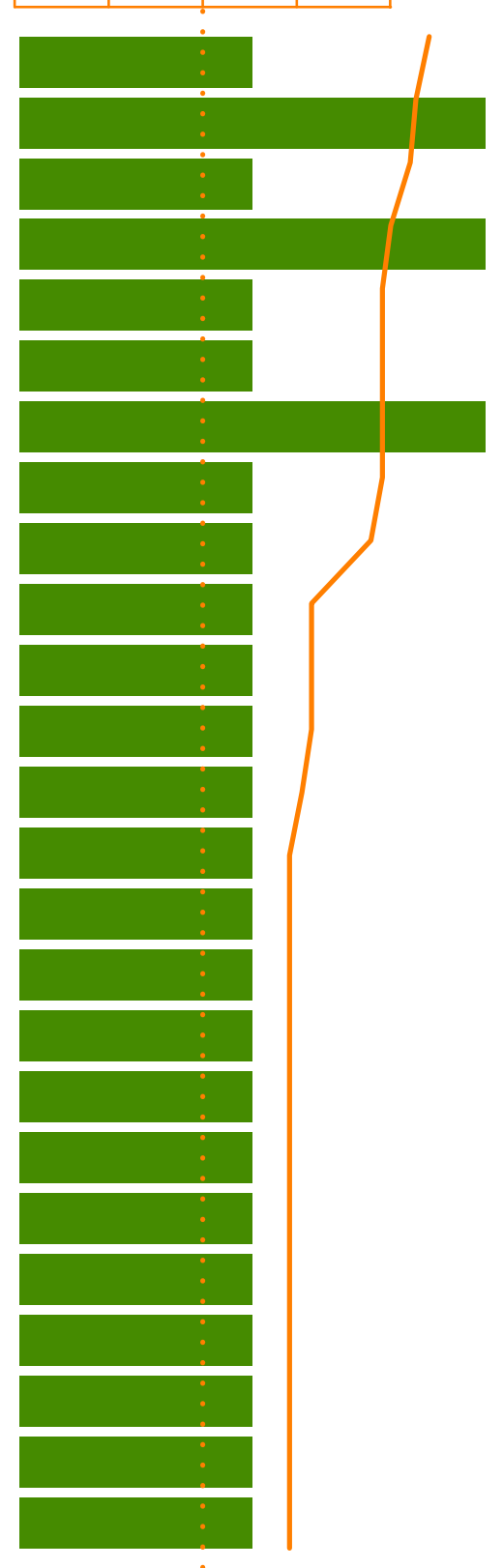

